\title{
GTPase HRas
}

National Cancer Institute

\section{Source}

National Cancer Institute. GTPase HRas. NCI Thesaurus. Code C17382.

GT Pase HRas (189 aa, 21 kDa) is encoded by the human HRAS gene. This protein plays a role in GT Pase activity and in signal transduction. 